\title{
Multiplicity of solutions for nonlinear impulsive differential equations with Dirichlet boundary conditions
}

\author{
Chenxing Zhou' ${ }^{1}$ Fenghua Miao ${ }^{1}$ and Sihua Liang ${ }^{1,2^{*}}$
}

\author{
"Correspondence: \\ liangsihua@163.com \\ ${ }^{1}$ College of Mathematics, \\ Changchun Normal University, \\ Changchun, Jilin 130032, P.R. China \\ ${ }^{2}$ Key Laboratory of Symbolic \\ Computation and Knowledge \\ Engineering of Ministry of \\ Education, Jilin University, \\ Changchun, 130012, P.R. China
}

\begin{abstract}
In this paper, we consider the existence of solutions for nonlinear impulsive differential equations with Dirichlet boundary conditions. Infinitely many solutions are obtained by using a version of the symmetric mountain-pass theorem, and this sequence of solutions converge to zero. Some recent results are extended.

MSC: $34 \mathrm{~B} 37 ; 35 \mathrm{~B} 38$
\end{abstract}

Keywords: impulsive effects; variational methods; Dirichlet boundary value problem; critical points

\section{Introduction}

In this paper, we study the following nonlinear impulsive differential equations with Dirichlet boundary conditions

$$
\left\{\begin{array}{l}
-u^{\prime \prime}(t)+a(t) u(t)=\mu|u|^{p-2} u(t)+f(t, u(t)), \quad \text { a.e. } t \in[0, T] \\
\Delta u^{\prime}\left(t_{j}\right)=u^{\prime}\left(t_{j}^{+}\right)-u^{\prime}\left(t_{j}^{-}\right)=I_{j}\left(u\left(t_{j}\right)\right), \quad j=1,2, \ldots, N \\
u(0)=u(T)=0
\end{array}\right.
$$

where $p>2, T>0, \mu>0, f:[0, T] \times \mathbb{R} \rightarrow \mathbb{R}$ is continuous, $a \in L^{\infty}[0, T], N$ is a positive integer, $0=t_{0}<t_{1}<t_{2}<\cdots<t_{N}<t_{N+1}=T, \Delta u^{\prime}\left(t_{j}\right)=u^{\prime}\left(t_{j}^{+}\right)-u^{\prime}\left(t_{j}^{-}\right)=\lim _{t \rightarrow t_{j}^{+}} u^{\prime}(t)-$ $\lim _{t \rightarrow t_{j}^{-}} u^{\prime}(t), I_{j}: \mathbb{R} \rightarrow \mathbb{R}$ are continuous. With the help of the symmetric mountain-pass lemma due to Kajikiya [1], we prove that there are infinitely many small weak solutions for equations (1.1) with the general nonlinearities $f(t, u)$.

In recent years, a great deal of works have been done in the study of the existence of solutions for impulsive boundary value problems, by which a number of chemotherapy, population dynamics, optimal control, ecology, industrial robotics and physics phenomena are described. For the general aspects of impulsive differential equations, we refer the reader to the classical monograph [2]. For some general and recent works on the theory of impulsive differential equations, we refer the reader to [3-13]. Some classical tools or techniques have been used to study such problems in the literature. These classical techniques include the coincidence degree theory [14], the method of upper and lower solutions with a monotone iterative technique [15], and some fixed point theorems in cones [16, 17]. 
On the other hand, in the last few years, many authors have used a variational method to study the existence and multiplicity of solutions for boundary value problems without impulsive effects [18-21]. For related basic information, we refer the reader to [22, 23].

For a second order differential equation $u^{\prime \prime}=f\left(t, u, u^{\prime}\right)$, one usually considers impulses in the position $u$ and the velocity $u^{\prime}$. However, in the motion of spacecraft, one has to consider instantaneous impulses depending on the position that result in jump discontinuities in velocity, but with no change in the position [24-27].

A new approach via critical point and variational methods is proved to be very effective in studying the boundary problem for differential equations. For some general and recent works on the theory of critical point theory and variational methods, we refer the reader to [28-37].

More precisely, in [28] the authors studied the following equations with Dirichlet boundary conditions:

$$
\left\{\begin{array}{l}
-\ddot{u}(t)+\lambda u(t)=f(t, u(t)), \quad \text { a.e. } t \in[0, T] \\
\Delta \dot{u}\left(t_{j}\right)=I_{j}\left(u\left(t_{j}\right)\right), \quad j=1,2, \ldots, p, \\
u(0)=u(T)=0 .
\end{array}\right.
$$

They obtained the existence of solutions for problems by using the variational method. Zhang and Yuan [30] extended the results in [28]. They obtained the existence of solutions for problem (1.2) with a perturbation term. Also, they obtained infinitely many solutions for problem (1.2) under the assumption that the nonlinearity $f$ is a superlinear case. Soon after that, Zhou and Li [29] extended problem (1.2). In all the above-mentioned works, the information on the sequence of solutions was not given.

Motivated by the fact above, the aim of this paper is to show the existence of infinitely many solutions for problem (1.1), and that there exists a sequence of infinitely many arbitrarily small solutions, converging to zero, by using a new version of the symmetric mountain-pass lemma due to Kajikiya [1]. Our main results extend the existing study.

Throughout this paper, we assume that $I_{j}: \mathbb{R} \rightarrow \mathbb{R}$ is continuous, and $f(t, u)$ satisfies the following conditions:

$\left(\mathrm{I}_{1}\right) \quad I_{j}(j=1,2, \ldots, N)$ are odd and satisfy

$$
\int_{0}^{u\left(t_{j}\right)} I_{j}(s) d s-\frac{1}{2} I_{j}\left(u\left(t_{j}\right)\right) u\left(t_{j}\right) \geq 0, \quad \int_{0}^{u\left(t_{j}\right)} I_{j}(s) d s \geq 0 ;
$$

( $\left.\mathrm{I}_{2}\right) \quad$ There exist $\delta_{j}>0, j=1,2, \ldots, N$ such that

$$
\int_{0}^{u\left(t_{j}\right)} I_{j}(s) d s \leq \delta_{j}|u|^{2}, \quad \text { for } u \in \mathbb{R} \backslash\{0\} ;
$$

$\left(\mathrm{H}_{1}\right) f(t, u) \in C([0, T] \times \mathbb{R}, \mathbb{R}), f(t,-u)=-f(t, u)$ for all $u \in \mathbb{R}$;

$\left(\mathrm{H}_{2}\right) \lim _{|u| \rightarrow \infty} \frac{f(t, u)}{\left.|u|\right|^{p-1}}=0$ uniformly for $t \in[0, T]$;

$\left(\mathrm{H}_{3}\right) \lim _{|u| \rightarrow 0^{+}} \frac{f(t, u)}{u}=\infty$ uniformly for $t \in[0, T]$.

The main result of this paper is as follows.

Theorem 1.1 Suppose that $\left(\mathrm{I}_{1}\right)-\left(\mathrm{I}_{2}\right)$ and $\left(\mathrm{H}_{1}\right)-\left(\mathrm{H}_{3}\right)$ hold. Then problem $(1.1)$ has a sequence of nontrivial solutions $\left\{u_{n}\right\}$ and $u_{n} \rightarrow 0$ as $n \rightarrow \infty$. 
Remark 1.1 Without the symmetry condition (i.e., $f(x,-u)=-f(x, u)$ and $I(-s)=-I(s)$ ), we can obtain at least one nontrivial solution by the same method in this paper.

Remark 1.2 We should point out that Theorem 1.1 is different from the previous results of [28-37] in three main directions:

(1) We do not make the nonlinearity $f$ satisfy the well-known Ambrosetti-Rabinowitz condition [23];

(2) We try to use Lusternik-Schnirelman's theory for $Z_{2}$-invariant functional. But since the functional is not bounded from below, we could not use the theory directly. So, we follow [38] to consider a truncated functional.

(3) We can obtain a sequence of nontrivial solutions $\left\{u_{n}\right\}$ and $u_{n} \rightarrow 0$ as $n \rightarrow \infty$.

Remark 1.3 There exist many functions $I_{j}$ and $f(t, u)$ satisfying conditions $\left(\mathrm{I}_{1}\right)-\left(\mathrm{I}_{2}\right)$ and $\left(\mathrm{H}_{1}\right)-\left(\mathrm{H}_{3}\right)$, respectively. For example, when $p=4, I_{j}(s)=s$ and $f(t, u)=e^{t} u^{1 / 3}$.

\section{Preliminary lemmas}

In this section, we first introduce some notations and some necessary definitions.

Definition 2.1 Let $E$ be a Banach space and $J: E \rightarrow \mathbb{R}$. $J$ is said to be sequentially weakly lower semi-continuous if $\lim _{n \rightarrow \infty} \inf J\left(u_{n}\right) \geq J(u)$ as $u_{n} \rightarrow u$ in $E$.

Definition 2.2 Let $E$ be a real Banach space. For any sequence $\left\{u_{n}\right\} \subset E$, if $\left\{J\left(u_{n}\right)\right\}$ is bounded and $J^{\prime}\left(u_{n}\right) \rightarrow 0$ as $n \rightarrow \infty$ possesses a convergent subsequence, then we say $J$ satisfies the Palais-Smale condition (denoted by $(P S)$ condition for short).

In the Sobolev space $H_{0}^{1}(0, T)$, consider the inner product

$$
\langle u, v\rangle_{H_{0}^{1}(0, T)}=\int_{0}^{T} u^{\prime}(t) v^{\prime}(t) d t,
$$

which induces the norm

$$
\|u\|_{H_{0}^{1}(0, T)}=\left(\int_{0}^{T}\left(u^{\prime}(t)\right)^{2} d t\right)^{\frac{1}{2}} .
$$

It is a consequence of Poincaré's inequality that

$$
\left(\int_{0}^{T}(u(t))^{2} d t\right)^{\frac{1}{2}} \leq \frac{1}{\sqrt{\lambda_{1}}}\left(\int_{0}^{T}\left(u^{\prime}(t)\right)^{2} d t\right)^{\frac{1}{2}} .
$$

Here, $\lambda_{1}=\pi^{2} / T^{2}$ is the first eigenvalue of the Dirichlet problem

$$
\left\{\begin{array}{l}
-u^{\prime \prime}(t)=\lambda u(t), \quad t \in[0, T] \\
u(0)=u(T)=0
\end{array}\right.
$$

In this paper, we will assume that $\inf _{t \in[0, T]} a(t)=m>-\lambda_{1}$. We can also define the inner product

$$
\langle u, v\rangle=\int_{0}^{T} u^{\prime}(t) v^{\prime}(t) d t+\int_{0}^{T} a(t) u(t) v(t) d t
$$


which induces the equivalent norm

$$
\|u\|=\left(\int_{0}^{T}\left(u^{\prime}(t)\right)^{2} d t+\int_{0}^{T} a(t)(u(t))^{2} d t\right)^{\frac{1}{2}}
$$

Lemma 2.1 [29] If $\operatorname{essinf}_{t \in[0, T]} a(t)=m>-\lambda_{1}$, then the norm $\|\cdot\|$ and the norm $\|\cdot\|_{H_{0}^{1}(0, T)}$ are equivalent.

Lemma 2.2 [29] There exists $c_{*}$ such that if $u \in H_{0}^{1}(0, T)$, then

$$
\|u\|_{\infty} \leq c_{*}\|u\|
$$

where $\|u\|_{\infty}=\max _{t \in[0, T]}|u(t)|$.

For $u \in H^{2}(0, T)$, we have that $u$ and $u^{\prime}$ are both absolutely continuous, and $u^{\prime \prime} \in L^{2}(0, T)$, hence, $\Delta u^{\prime}\left(t_{j}\right)=u^{\prime}\left(t_{j}^{+}\right)-u^{\prime}\left(t_{j}^{-}\right)$for any $t \in[0, T]$. If $u \in H_{0}^{1}(0, T)$, then $u$ is absolutely continuous and $u^{\prime} \in L^{2}(0, T)$. In this case, the one-side derivatives $u^{\prime}\left(t_{j}^{+}\right)$and $u^{\prime}\left(t_{j}^{-}\right)$may not exist. As a consequence, we need to introduce a different concept of solution. Suppose that $u \in C[0, T]$ satisfies the Dirichlet condition $u(0)=u(T)=0$. Assume that, for every $j=1,2, \ldots, N, u_{j}=\left.u\right|_{\left(t_{j}, t_{j+1}\right)}$ and $u_{j} \in H^{2}\left(t_{j}, t_{j+1}\right)$. Let $0=t_{0}<t_{1}<t_{2}<\cdots<t_{N}<t_{N+1}=T$.

Taking $v \in H_{0}^{1}(0, T)$ and multiplying the two sides of the equality

$$
-u^{\prime \prime}(t)+a(t) u(t)=\mu|u|^{p-2} u(t)+f(t, u(t))
$$

by $v$ and integrating between 0 and $T$, we have

$$
\int_{0}^{T}\left[-u^{\prime \prime}(t)+a(t) u(t)-\mu|u|^{p-2} u(t)-f(t, u(t))\right] v(t) d t=0 .
$$

Moreover, since $u(0)=u(T)=0$, one has

$$
\begin{aligned}
& -\int_{0}^{T} u^{\prime \prime}(t) v(t) d t \\
& =-\sum_{j=0}^{N} \int_{t_{j}}^{t_{j+1}} u^{\prime \prime}(t) v(t) d t \\
& =-\left.\sum_{j=0}^{N} u^{\prime}(t) v(t)\right|_{t_{j}^{+}} ^{t_{j+1}^{-}}+\int_{0}^{T} u^{\prime}(t) v^{\prime}(t) d t \\
& =-\left(-\sum_{j=1}^{N} \Delta u^{\prime}\left(t_{j}\right) v\left(t_{j}\right)-u^{\prime}(0) v(0)+u^{\prime}(T) v(T)\right)+\int_{0}^{T} u^{\prime}(t) v^{\prime}(t) d t \\
& =\sum_{j=1}^{N} \Delta u^{\prime}\left(t_{j}\right) v\left(t_{j}\right)+\int_{0}^{T} u^{\prime}(t) v^{\prime}(t) d t \\
& =\sum_{j=1}^{N} I_{j}\left(u\left(t_{j}\right)\right) v\left(t_{j}\right)+\int_{0}^{T} u^{\prime}(t) v^{\prime}(t) d t .
\end{aligned}
$$


Combining (2.3), we get

$$
\begin{gathered}
\int_{0}^{T} u^{\prime}(t) v^{\prime}(t) d t+\int_{0}^{T} a(t) u(t) v(t) d t-\mu \int_{0}^{T}|u|^{p-2} u(t) v(t) d t \\
-\int_{0}^{T} f(t, u(t)) v(t) d t+\sum_{j=1}^{N} I_{j}\left(u\left(t_{j}\right)\right) v\left(t_{j}\right)=0 .
\end{gathered}
$$

Lemma 2.3 A weak solution of (1.1) is a function $u \in H_{0}^{1}(0, T)$ such that

$$
\begin{gathered}
\int_{0}^{T} u^{\prime}(t) v^{\prime}(t) d t+\int_{0}^{T} a(t) u(t) v(t) d t-\mu \int_{0}^{T}|u|^{p-2} u(t) v(t) d t \\
-\int_{0}^{T} f(t, u(t)) v(t) d t+\sum_{j=1}^{N} I_{j}\left(u\left(t_{j}\right)\right) v\left(t_{j}\right)=0
\end{gathered}
$$

for any $v \in H_{0}^{1}(0, T)$.

$$
\text { Consider } J: H_{0}^{1}(0, T) \rightarrow \mathbb{R} \text { defined by }
$$

$$
J(u)=\frac{1}{2}\|u\|^{2}-\frac{\mu}{p} \int_{0}^{T}|u|^{p} d t-\int_{0}^{T} F(t, u(t)) d t+\sum_{j=1}^{N} \int_{0}^{u\left(t_{j}\right)} I_{j}(s) d s
$$

where $F(t, u)=\int_{0}^{u} f(t, s) d s$. Using the continuity of $f$ and $I_{j}, j=1,2, \ldots, N$, we obtain the continuity and differentiability of $J$ and $J \in C^{1}\left(H_{0}^{1}(0, T), \mathbb{R}\right)$. For any $v \in H_{0}^{1}(0, T)$, one has

$$
\begin{aligned}
J^{\prime}(u) v= & \int_{0}^{T} u^{\prime}(t) v^{\prime}(t) d t+\int_{0}^{T} a(t) u(t) v(t) d t \\
& -\mu \int_{0}^{T}|u|^{p-2} u(t) v(t) d t \\
& -\int_{0}^{T} f(t, u(t)) v(t) d t+\sum_{j=1}^{N} I_{j}\left(u\left(t_{j}\right)\right) v\left(t_{j}\right) .
\end{aligned}
$$

Thus, the solutions of problem (1.1) are the corresponding critical points of $J$.

Lemma 2.4 If $u \in H_{0}^{1}(0, T)$ is a weak solution of problem (1.1), then $u$ is a classical solution of problem (1.1).

Proof Obviously, we have $u(0)=u(T)=0$ since $u \in H_{0}^{1}(0, T)$. By the definition of weak solution, for any $v \in H_{0}^{1}(0, T)$, one has

$$
\begin{gathered}
\int_{0}^{T} u^{\prime}(t) v^{\prime}(t) d t+\int_{0}^{T} a(t) u(t) v(t) d t-\mu \int_{0}^{T}|u|^{p-2} u(t) v(t) d t \\
-\int_{0}^{T} f(t, u(t)) v(t) d t+\sum_{j=1}^{N} I_{j}\left(u\left(t_{j}\right)\right) v\left(t_{j}\right)=0 .
\end{gathered}
$$


For $j \in\{0,1,2, \ldots, N\}$, choose $v \in H_{0}^{1}(0, T)$ with $v(t)=0$ for every $t \in\left[0, t_{j}\right] \cup\left[t_{j+1}, T\right]$. Then

$$
\begin{aligned}
& \int_{t_{j}}^{t_{j+1}} u^{\prime}(t) v^{\prime}(t) d t+\int_{t_{j}}^{t_{j+1}} a(t) u(t) v(t) d t \\
& \quad=\mu \int_{t_{j}}^{t_{j+1}}|u|^{p-2} u(t) v(t) d t+\int_{0}^{T} f(t, u(t)) v(t) d t
\end{aligned}
$$

By the definition of weak derivative, the equality above implies that

$$
-u^{\prime \prime}(t)+a(t) u(t)=\mu|u|^{p-2} u(t)+f(t, u(t)), \quad \text { a.e. } t \in\left(t_{j}, t_{j+1}\right)
$$

Hence $u_{j} \in H^{2}\left(t_{j}, t_{j+1}\right)$ and $u$ satisfies the equation in (1.1) a.e. on $[0, T]$. By integrating (2.7), we have

$$
\begin{aligned}
& -\sum_{j=1}^{N} \Delta u^{\prime}\left(t_{j}\right) v\left(t_{j}\right)+u^{\prime}(T) v(T)-u^{\prime}(0) v(0)+\sum_{j=1}^{N} I_{j}\left(u\left(t_{j}\right)\right) v\left(t_{j}\right) \\
& \quad+\int_{0}^{T}\left[-u^{\prime \prime}(t)+a(t) u(t)-\mu|u|^{p-2} u(t)-f(t, u(t))\right] v(t) d t=0 .
\end{aligned}
$$

Combining this fact with (2.8), we get

$$
\sum_{j=1}^{N} \Delta u^{\prime}\left(t_{j}\right) v\left(t_{j}\right)=\sum_{j=1}^{N} I_{j}\left(u\left(t_{j}\right)\right) v\left(t_{j}\right) \quad \text { for any } v \in H_{0}^{1}(0, T) .
$$

Hence, $\Delta u^{\prime}\left(t_{j}\right)=I_{j}\left(u\left(t_{j}\right)\right)$ for every $j=1,2, \ldots, N$, and the impulsive condition in (1.1) is satisfied. This completes the proof.

Lemma 2.5 If $\operatorname{ess~inf~}_{t \in[0, T]} a(t)=m>-\lambda_{1}$, then the functional J is sequentially weakly lower semi-continuous.

Proof Let $\left\{u_{n}\right\}$ be a weakly convergent sequence to $u$ in $H_{0}^{1}(0, T)$, then

$$
\|u\| \leq \lim _{n \rightarrow \infty} \inf \left\|u_{n}\right\|
$$

We have that $\left\{u_{n}\right\}$ converges uniformly to $u$ on $C[0, T]$. Then

$$
\begin{aligned}
& \lim _{n \rightarrow \infty} \inf J\left(u_{n}\right) \\
& =\lim _{n \rightarrow \infty}\left[\frac{1}{2}\left\|u_{n}\right\|^{2}-\frac{\mu}{p} \int_{0}^{T}\left|u_{n}\right|^{p} d t-\int_{0}^{T} F\left(t, u_{n}(t)\right) d t+\sum_{j=1}^{N} \int_{0}^{u_{n}\left(t_{j}\right)} I_{j}(s) d s\right] \\
& \geq \frac{1}{2}\|u\|^{2}-\frac{\mu}{p} \int_{0}^{T}|u|^{p} d t-\int_{0}^{T} F(t, u(t)) d t+\sum_{j=1}^{N} \int_{0}^{u\left(t_{j}\right)} I_{j}(s) d s \\
& \quad=J(u) .
\end{aligned}
$$

This completes the proof. 
Under assumptions $\left(\mathrm{H}_{1}\right)$ and $\left(\mathrm{H}_{2}\right)$, we have

$$
\begin{aligned}
& f(t, u) u=o\left(|u|^{p}\right), \\
& F(t, u)=o\left(|u|^{p}\right) \quad \text { as }|u| \rightarrow \infty,
\end{aligned}
$$

which means that for all $\varepsilon>0$, there exist $a(\varepsilon), b(\varepsilon)>0$ such that

$$
\begin{aligned}
& |f(t, u) u| \leq a(\varepsilon)+\varepsilon|u|^{p}, \\
& |F(x, u)| \leq b(\varepsilon)+\varepsilon|u|^{p} .
\end{aligned}
$$

Hence, for every positive constant $k$, we have

$$
F(x, u)-k f(x, u) u \leq c(\varepsilon)+\varepsilon|u|^{p},
$$

where $c(\varepsilon)>0$.

Lemma 2.6 Suppose that $\left(\mathrm{I}_{1}\right)-\left(\mathrm{I}_{2}\right)$ and $\left(\mathrm{H}_{1}\right)-\left(\mathrm{H}_{3}\right)$ hold, then $J(u)$ satisfies the $(P S)$ condition.

Proof Let $\left\{u_{n}\right\}$ be a sequence in $H_{0}^{1}(0, T)$ such that $\left\{J\left(u_{n}\right)\right\}$ is bounded and $J^{\prime}\left(u_{n}\right) \rightarrow 0$ as $n \rightarrow \infty$. First, we prove that $\left\{u_{n}\right\}$ is bounded. By (2.5), (2.6) and (2.11), one has

$$
\begin{aligned}
J\left(u_{n}\right) & -\frac{1}{2} J^{\prime}\left(u_{n}\right) u_{n} \\
= & \left(\frac{1}{2}-\frac{1}{p}\right) \mu \int_{0}^{T}\left|u_{n}\right|^{p} d t+\int_{0}^{T}\left[\frac{1}{2} f\left(t, u_{n}(t)\right) u_{n}(t)-F\left(t, u_{n}(t)\right)\right] d t \\
& +\sum_{j=1}^{N} \int_{0}^{u_{n}\left(t_{j}\right)} I_{j}(s) d s-\frac{1}{2} \sum_{j=1}^{N} I_{j}\left(u_{n}\left(t_{j}\right)\right) u_{n}\left(t_{j}\right) \\
\geq & \left(\frac{(p-2) \mu}{2 p}-\varepsilon\right) \int_{0}^{T}\left|u_{n}\right|^{p} d t-c(\varepsilon) T+\sum_{j=1}^{N} \int_{0}^{u_{n}\left(t_{j}\right)} I_{j}(s) d s \\
& -\frac{1}{2} \sum_{j=1}^{N} I_{j}\left(u_{n}\left(t_{j}\right)\right) u_{n}\left(t_{j}\right) .
\end{aligned}
$$

By condition $\left(\mathrm{I}_{1}\right)$, we can deduce that

$$
\sum_{j=1}^{N} \int_{0}^{u_{n}\left(t_{j}\right)} I_{j}(s) d s-\frac{1}{2} \sum_{j=1}^{N} I_{j}\left(u_{n}\left(t_{j}\right)\right) u_{n}\left(t_{j}\right) \geq 0 .
$$

Setting $\varepsilon=\frac{(p-2) \mu}{4 p}$, we get

$$
\int_{0}^{T}\left|u_{n}\right|^{p} d t \leq M+o(1)\left\|u_{n}\right\|
$$


where $o(1) \rightarrow 0$ and $M$ is a positive constant. On the other hand, by $\left(\mathrm{I}_{1}\right),(2.5)$ and (2.10), we have

$$
\begin{aligned}
\infty & >J\left(u_{n}\right)=\frac{1}{2}\left\|u_{n}\right\|^{2}-\frac{\mu}{p} \int_{0}^{T}\left|u_{n}\right|^{p} d t-\int_{0}^{T} F\left(t, u_{n}(t)\right) d t+\sum_{j=1}^{N} \int_{0}^{u_{n}\left(t_{j}\right)} I_{j}(s) d s \\
& \geq \frac{1}{2}\left\|u_{n}\right\|^{2}-\left(\frac{\mu}{p}+\varepsilon\right) \int_{0}^{T}\left|u_{n}\right|^{p} d t-b(\varepsilon) T .
\end{aligned}
$$

Thus, (2.12) and (2.13) imply that $\left\{u_{n}\right\}$ is bounded in $H_{0}^{1}(0, T)$. Going if necessary to a subsequence, we can assume that there exists $u \in H_{0}^{1}(0, T)$ such that

$$
\begin{array}{ll}
u_{n} \rightarrow u & \text { weakly in } H_{0}^{1}(0, T), \\
u_{n} \rightarrow u & \text { strongly in } C([0, T], \mathbb{R}),
\end{array}
$$

as $n \rightarrow \infty$. Hence,

$$
\begin{aligned}
& \left(J^{\prime}\left(u_{n}\right)-J^{\prime}(u)\right)\left(u_{n}-u\right) \rightarrow 0, \\
& \int_{0}^{T}\left[f\left(t, u_{n}(t)\right)-f(t, u(t))\right]\left(u_{n}(t)-u(t)\right) d t \rightarrow 0, \\
& \int_{0}^{T}\left(\left|u_{n}\right|^{p-2} u_{n}(t)-|u|^{p-2} u(t)\right)\left(u_{n}(t)-u(t)\right) d t \rightarrow 0, \\
& \sum_{j=1}^{N}\left[I_{j}\left(u_{n}\left(t_{j}\right)\right)-I_{j}\left(u\left(t_{j}\right)\right)\right]\left(u_{n}\left(t_{j}\right)-u\left(t_{j}\right)\right) \rightarrow 0,
\end{aligned}
$$

as $n \rightarrow \infty$. Moreover, one has

$$
\begin{aligned}
\left(J^{\prime}\left(u_{n}\right)-J^{\prime}(u)\right)\left(u_{n}-u\right)= & \left\|u_{n}-u\right\|^{2}-\int_{0}^{T}\left(\left|u_{n}\right|^{p-2} u_{n}(t)-|u|^{p-2} u(t)\right)\left(u_{n}(t)-u(t)\right) d t \\
& -\int_{0}^{T}\left[f\left(t, u_{n}(t)\right)-f(t, u(t))\right]\left(u_{n}(t)-u(t)\right) d t \\
& -\sum_{j=1}^{N}\left[I_{j}\left(u_{n}\left(t_{j}\right)\right)-I_{j}\left(u\left(t_{j}\right)\right)\right]\left(u_{n}\left(t_{j}\right)-u\left(t_{j}\right)\right) .
\end{aligned}
$$

Therefore, $\left\|u_{n}-u\right\| \rightarrow 0$ as $n \rightarrow+\infty$. That is $\left\{u_{n}\right\}$ converges strongly to $u$ in $H_{0}^{1}(0, T)$. That is $J$ satisfies the $(P S)$ condition.

\section{Existence of a sequence of arbitrarily small solutions}

In this section, we prove the existence of infinitely many solutions of (1.1), which tend to zero. Let $X$ be a Banach space and denote

$$
\Sigma:=\{A \subset X \backslash\{0\}: A \text { is closed in } X \text { and symmetric with respect to the orgin }\} .
$$

For $A \in \Sigma$, we define genus $\gamma(A)$ as

$$
\gamma(A):=\inf \left\{m \in N: \exists \varphi \in C\left(A, R^{m} \backslash\{0\},-\varphi(x)=\varphi(-x)\right)\right\} .
$$


If there is no mapping $\varphi$ as above for any $m \in N$, then $\gamma(A)=+\infty$. We list some properties of the genus (see [1]).

Proposition 3.1 Let $A$ and $B$ be closed symmetric subsets of $X$, which do not contain the origin. Then the following hold.

(1) If there exists an odd continuous mapping from $A$ to $B$, then $\gamma(A) \leq \gamma(B)$;

(2) If there is an odd homeomorphism from $A$ to $B$, then $\gamma(A)=\gamma(B)$;

(3) If $\gamma(B)<\infty$, then $\gamma \overline{(A \backslash B)} \geq \gamma(A)-\gamma(B)$;

(4) Then $n$-dimensional sphere $S^{n}$ has a genus of $n+1$ by the Borsuk-Ulam theorem;

(5) If $A$ is compact, then $\gamma(A)<+\infty$ and there exists $\delta>0$ such that $U_{\delta}(A) \in \Sigma$ and $\gamma\left(U_{\delta}(A)\right)=\gamma(A)$, where $U_{\delta}(A)=\{x \in X:\|x-A\| \leq \delta\}$.

Let $\Sigma_{k}$ denote the family of closed symmetric subsets $A$ of $X$ such that $0 \notin A$ and $\gamma(A) \geq k$. The following version of the symmetric mountain-pass lemma is due to Kajikiya $[1]$.

Lemma 3.1 Let $E$ be an infinite-dimensional space and $I \in C^{1}(E, R)$, and suppose the following conditions hold.

$\left(C_{1}\right) I(u)$ is even, bounded from below, $I(0)=0$ and $I(u)$ satisfies the Palais-Smale condition;

$\left(C_{2}\right)$ For each $k \in N$, there exists an $A_{k} \in \Sigma_{k}$ such that $\sup _{u \in A_{k}} I(u)<0$.

Then either $\left(\mathrm{R}_{1}\right)$ or $\left(\mathrm{R}_{2}\right)$ below holds.

$\left(R_{1}\right)$ There exists a sequence $\left\{u_{k}\right\}$ such that $I^{\prime}\left(u_{k}\right)=0, I\left(u_{k}\right)<0$ and $\left\{u_{k}\right\}$ converges to zero;

$\left(\mathrm{R}_{2}\right)$ There exist two sequences $\left\{u_{k}\right\}$ and $\left\{v_{k}\right\}$ such that $I^{\prime}\left(u_{k}\right)=0, I\left(u_{k}\right)<0, u_{k} \neq 0$, $\lim _{k \rightarrow \infty} u_{k}=0, I^{\prime}\left(v_{k}\right)=0, I\left(v_{k}\right)<0, \lim _{k \rightarrow \infty} v_{k}=0$, and $\left\{v_{k}\right\}$ converges to a nonzero limit.

Remark 3.1 From Lemma 3.1, we have a sequence $\left\{u_{k}\right\}$ of critical points such that $I\left(u_{k}\right) \leq$ $0, u_{k} \neq 0$ and $\lim _{k \rightarrow \infty} u_{k}=0$.

In order to get infinitely many solutions, we need some lemmas. Under the assumptions of Theorem 1.1, let $\varepsilon=\frac{\mu}{p}$, we have

$$
\begin{aligned}
J(u) & =\frac{1}{2}\|u\|^{2}-\frac{\mu}{p} \int_{0}^{T}|u|^{p} d t-\int_{0}^{T} F(t, u(t)) d t+\sum_{j=1}^{N} \int_{0}^{u\left(t_{j}\right)} I_{j}(s) d s \\
& \geq \frac{1}{2}\|u\|^{2}-\left(\frac{\mu}{p}+\varepsilon\right) \int_{0}^{T}|u|^{p} d t-b(\varepsilon) T \\
& \geq \frac{1}{2}\|u\|^{2}-\left(\frac{\mu}{p}+\varepsilon\right) c_{*}^{p} T\|u\|^{p}-b(\varepsilon) T \\
& =\frac{1}{2}\|u\|^{2}-\frac{2 \mu}{p} c_{*}^{p} T\|u\|^{p}-b\left(\frac{\mu}{p}\right) T \\
& =A\|u\|^{2}-B\|u\|^{p}-C,
\end{aligned}
$$

where

$$
A=\frac{1}{2}, \quad B=\frac{2 \mu}{p} c_{*}^{p} T, \quad C=b\left(\frac{\mu}{p}\right) T .
$$


Let $P(t)=A t^{2}-B t^{p}-C$. As $P(s)$ attains a local but not a global minimum ( $P$ is not bounded below), we have to perform some sort of truncation. To this end, let $R_{0}, R_{1}$ be such that $m<R_{0}<M<R_{1}$, where $m$ is the local minimum of $P(s)$, and $M$ is the local maximum and $P\left(R_{1}\right)>P(m)$. For these values $R_{1}$ and $R_{0}$, we can choose a smooth function $\chi(t)$ defined as follows

$$
\chi(t)= \begin{cases}1, & 0 \leq t \leq R_{0} \\ 0, & t \geq R_{1}, \\ C^{\infty}, \chi(t) \in[0,1], & R_{0} \leq t \leq R_{1} .\end{cases}
$$

Then it is easy to see $\chi(t) \in[0,1]$ and $\chi(t)$ is $C^{\infty}$. Let $\varphi(u)=\chi(\|u\|)$ and consider the perturbation of $J(u)$ :

$$
\begin{aligned}
G(u)= & \frac{1}{2}\|u\|^{2}-\frac{\varphi(u) \mu}{p} \int_{0}^{T}|u|^{p} d t \\
& -\varphi(u) \int_{0}^{T} F(t, u(t)) d t+\sum_{j=1}^{N} \int_{0}^{u\left(t_{j}\right)} I_{j}(s) d s .
\end{aligned}
$$

Then

$$
\begin{aligned}
G(u) & \geq A\|u\|^{2}-B \varphi(u)\|u\|^{p}-C \\
& =\bar{P}(\|u\|),
\end{aligned}
$$

where $\bar{P}(t)=A t^{2}-B \chi(t) t^{p}-C$ and

$$
\bar{P}(t)= \begin{cases}P(t), & 0 \leq t \leq \rho_{0}, \\ m^{*}, & t \geq \rho_{1} .\end{cases}
$$

From the arguments above, we have the following.

Lemma 3.2 Let $G(u)$ is defined as in (3.1). Then

(i) $G \in C^{1}\left(H_{0}^{1}(0, T), \mathbb{R}\right)$ and $G$ is even and bounded from below;

(ii) If $G(u)<m$, then $\bar{P}(\|u\|)<m$, consequently, $\|u\|<\rho_{0}$ and $J(u)=G(u)$;

(iii) Suppose that $\left(\mathrm{I}_{1}\right)-\left(\mathrm{I}_{2}\right)$ and $\left(\mathrm{H}_{1}\right)-\left(\mathrm{H}_{3}\right)$ hold, then $G(u)$ satisfies the $(P S)$ condition.

Proof It is easy to see (i) and (ii). (iii) are consequences of (ii) and Lemma 2.6.

Lemma 3.3 Assume that $\left(\mathrm{I}_{2}\right)$ and $\left(\mathrm{H}_{3}\right)$ hold. Then for any $k \in N$, there exists $\delta=\delta(k)>0$ such that $\gamma\left(\left\{u \in H_{0}^{1}(0, T): G(u) \leq-\delta(k)\right\} \backslash\{0\}\right) \geq k$.

Proof Firstly, by $\left(\mathrm{H}_{3}\right)$ of Theorem 1.1, for any fixed $u \in H_{0}^{1}(0, T), u \neq 0$, we have

$$
F(x, \rho u) \geq M(\rho)(\rho u)^{2} \quad \text { with } M(\rho) \rightarrow \infty \text { as } \rho \rightarrow 0 .
$$

Secondly, from Lemma 5 of [33], we have that for any finite dimensional subspace $E_{k}$ of $H_{0}^{1}(0, T)$ and any $u \in E_{k}$, there exists a constant $d>0$ such that

$$
|u|_{s}=\left(\int_{0}^{T}|u(t)|^{s} d t\right)^{\frac{1}{s}} \geq d\|u\|, \quad s \geq 1, \forall u \in E_{k}
$$


Therefore, for any $u \in E_{k}$ with $\|u\|=1$ and $\rho$ small enough, we have

$$
\begin{aligned}
G(\rho u) & =J(\rho u) \leq \frac{1}{2} \rho^{2}-\frac{\mu}{p} \rho^{p} \int_{0}^{T}|u|^{p} d t-M(\rho) \rho^{2} \int_{0}^{T}|u|^{2} d t+\rho^{2} \sum_{j=1}^{N} \delta_{j} c_{*}^{2} \\
& \leq\left(\frac{1}{2}+\sum_{j=1}^{N} \delta_{j} c_{*}^{2}-M(\rho) d^{2}\right) \rho^{2} \\
& =-\delta(k)<0,
\end{aligned}
$$

since $\lim _{|\rho| \rightarrow 0} M(\rho)=+\infty$. That is,

$$
\left\{u \in E_{k}:\|u\|=\rho\right\} \subset\left\{u \in H_{0}^{1}(0, T): G(u) \leq-\delta(k)\right\} \backslash\{0\} .
$$

This completes the proof.

Now, we give the proof of Theorem 1.1 as following.

Proof of Theorem 1.1 Recall that

$$
\Sigma_{k}=\left\{A \in H_{0}^{1}(0, T) \backslash\{0\}: A \text { is closed and } A=-A, \gamma(A) \geq k\right\}
$$

and define

$$
c_{k}=\inf _{A \in \Sigma_{k}} \sup _{u \in A} G(u)
$$

By Lemma 3.2(i) and Lemma 3.3, we know that $-\infty<c_{k}<0$. Therefore, assumptions $\left(C_{1}\right)$ and $\left(C_{2}\right)$ of Lemma 3.1 are satisfied. This means that $G$ has a sequence of solutions $\left\{u_{n}\right\}$ converging to zero. Hence, Theorem 1.1 follows by Lemma 3.2(ii).

\section{Competing interests}

The authors declare that they have no competing interests.

\section{Authors' contributions}

CZ carried out the theoretical studies, and participated in the sequence alignment and drafted the manuscript. FM participated in the design of the study and performed the statistical analysis. SL conceived of the study, and participated in its design and coordination. All authors read and approved the final manuscript.

\section{Author details}

${ }^{1}$ College of Mathematics, Changchun Normal University, Changchun, Jilin 130032, P.R. China. ${ }^{2}$ Key Laboratory of Symbolic Computation and Knowledge Engineering of Ministry of Education, Jilin University, Changchun, 130012, P.R. China.

\section{Acknowledgements}

The authors are supported by the Research Foundation during the 12th Five-Year Plan Period of Department of Education of Jilin Province, China (Grant [2013] No. 252), the China Postdoctoral Science Foundation (Grant No. 2012M520665), the Youth Foundation for Science and Technology Department of Jilin Province (20130522100JH), the open project program of Key Laboratory of Symbolic Computation and Knowledge Engineering of Ministry of Education, Jilin University (Grant No. 93K172013K03), the Natural Science Foundation of Changchun Normal University. 


\section{References}

1. Kajikiya, R: A critical-point theorem related to the symmetric mountain-pass lemma and its applications to elliptic equations. J. Funct. Anal. 225, 352-370 (2005)

2. Lakshmikantham, V, Bainov, DD, Simeonov, PS: Theory of Impulsive Differential Equations. World Scientific, Singapore (1989)

3. Agarwal, RP, Franco, D, O'Regan, D: Singular boundary value problems for first and second order impulsive differential equations. Aequ. Math. 69, 83-96 (2005)

4. Nieto, JJ, Rodriquez-Lopez, R: Boundary value problems for a class of impulsive functional equations. Comput. Math. Appl. 55, 2715-2731 (2008)

5. Li, J, Nieto, JJ, Shen, J: Impulsive periodic boundary value problems of first-order differential equations. J. Math. Anal. Appl. 325, 226-299 (2007)

6. Nieto, JJ, Rodriguez-Lopez, R: New comparison results for impulsive integro-differential equations and applications. J. Math. Anal. Appl. 328, 1343-1368 (2007)

7. Samoilenko, AM, Perestyuk, NA: Impulsive Differential Equations. World Scientific, Singapore (1995)

8. Zhang, H, Chen, L, Nieto, JJ: A delayed epidemic model with stage structure and pulses for management strategy. Nonlinear Anal., Real World Appl. 9, 1714-1726 (2008)

9. Benchohra, M, Henderson, J, Ntouyas, SK: Impulsive Differential Equations and Inclusions, vol. 2. Hindawi Publishing Corporation, New York (2006)

10. Zeng, G, Wang, F, Nieto, J: Complexity of a delayed predator-prey model with impulsive harvest and Holling-type II functional response. Adv. Complex Syst. 11, 77-97 (2008)

11. Cardinali, T, Servadei, R: Existence theorems for periodic semilinear impulsive problems (viable and not viable cases). Pure Math. Appl. 14(3), 161-171 (2003)

12. Cardinali, T, Servadei, R: Periodic solutions of nonlinear impulsive differential inclusions with constraints. Proc. Am. Math. Soc. 132(8), 2339-2349 (2004)

13. Cardinali, T, Servadei, R: On the existence of solutions for nonlinear impulsive periodic viable problems. Cent. Eur. J. Math. 2(4), 573-583 (2004)

14. Qian, D, Li, X: Periodic solutions for ordinary differential equations with sublinear impulsive effects. J. Math. Anal. Appl. 303, 288-303 (2005)

15. Chen, L, Sun, J: Nonlinear boundary value problem for first order impulsive functional differential equations. J. Math. Anal. Appl. 318, 726-741 (2006)

16. Chen, L, Tisdel, CC, Yuan, R: On the solvability of periodic boundary value problems with impulse. J. Math. Anal. Appl. $331,233-244(2007)$

17. Chu, J, Nieto, JJ: Impulsive periodic solution of first-order singular differential equations. Bull. Lond. Math. Soc. 40 , $143-150(2008)$

18. He, XM, Zou, WM: Infinitely many arbitrarily small solutions for singular elliptic problems with critical Sobolev-Hardy exponents. Proc. Edinb. Math. Soc. 52, 97-108 (2009)

19. Chen, J, Li, S: On multiple solutions of a singular quasi-linear equation on unbounded domain. J. Math. Anal. Appl. 275, 733-746 (2002)

20. Brézis, H, Nirenberg, L: Positive solutions of nonlinear elliptic equations involving critical exponents. Commun. Pure Appl. Math. 34, 437-477 (1983)

21. Li, S, Zou, W: Remarks on a class of elliptic problems with critical exponents. Nonlinear Anal. 32, 769-774 (1998)

22. Mawhin, J, Willem, M: Critical Point Theory and Hamiltonian Systems. Springer, Berlin (1989)

23. Rabinowitz, PH: Minimax Methods in Critical Point Theory with Applications to Differential Equations. CBMS Regional Conf. Ser. in. Math., vol. 65. Am. Math. Soc., Providence (1986)

24. Carter, TE: Optimal impulsive space trajectories based on linear equations. J. Optim. Theory Appl. 70, 277-297 (1991)

25. Carter, TE: Necessary and sufficient conditions for optimal impulsive rendezvous with linear equations of motion Dyn. Control 10, 219-227 (2000)

26. Liu, X, Willms, AR: Impulsive controllability of linear dynamical systems with applications to maneuvers of spacecraft. Math. Probl. Eng. 2, 277-299 (1996)

27. Prado, AFBA: Bi-impulsive control to build a satellite constellation. Nonlinear Dyn. Syst. Theory 5, $169-175$ (2005)

28. Nieto, JJ, O’Regan, D: Variational approach to impulsive differential equations. Nonlinear Anal., Real World Appl. 10, 680-690 (2009)

29. Zhou, J, Li, Y: Existence and multiplicity of solutions for some Dirichlet problems with impulse effects. Nonlinear Anal. TMA 71, 2856-2865 (2009)

30. Zhang, Z, Yuan, R: An application of variational methods to Dirichlet boundary value problem with impulses. Nonlinear Anal., Real World Appl. 11, 155-162 (2010)

31. Sun, J, Chen, H: Multiplicity of solutions for a class of impulsive differential equations with Dirichlet boundary conditions via variant fountain theorems. Nonlinear Anal., Real World Appl. 11, 4062-4071 (2010)

32. Sun, J, Chen, H, Nieto, JJ, Otero-Novoa, M: Multiplicity of solutions for perturbed second-order Hamiltonian systems with impulsive effects. Nonlinear Anal. TMA 72, 4575-4586 (2010)

33. Zhang, $\mathrm{H}$, Li, Z: Variational approach to impulsive differential equations with periodic boundary conditions. Nonlinear Anal., Real World Appl. 11, 67-78 (2010)

34. Tian, Y, Ge, W: Variational methods to Sturm-Liouville boundary value problem for impulsive differential equations. Nonlinear Anal. TMA 72, 277-287 (2010)

35. Nieto, JJ: Variational formulation of a damped Dirichlet impulsive problem. Appl. Math. Lett. 23, 940-942 (2010)

36. Li, W-S, Chang, Y-K, Nieto, JJ: Solvability of impulsive neutral evolution differential inclusions with state-dependent delay. Math. Comput. Model. 49, 1920-1927 (2009)

37. Zhang, D, Bai, BX: Existence of solutions for nonlinear impulsive differential equations with Dirichlet boundary conditions. Math. Comput. Model. 53, 1154-1161 (2011)

38. Garcia Azorero, J, Peral Alonso, I: Multiplicity of solutions for elliptic problems with critical exponent or with a nonsymmetric term. Trans. Am. Math. Soc. 323, 877-895 (1991) 
doi:10.1186/1687-2770-2013-200

Cite this article as: Zhou et al.: Multiplicity of solutions for nonlinear impulsive differential equations with Dirichlet boundary conditions. Boundary Value Problems 2013 2013:200.

Submit your manuscript to a SpringerOpen ${ }^{\circ}$ journal and benefit from:

- Convenient online submission

- Rigorous peer review

- Immediate publication on acceptance

- Open access: articles freely available online

- High visibility within the field

- Retaining the copyright to your article

Submit your next manuscript at $\gg$ springeropen.com 